\title{
AN HR DIAGRAM FOR THE LMC FROM THE MEDIUM DEEP
}

\section{SURVEY}

DUNCAN A. FORBES

Lick Observatory, University of California, Santa Cruz, CA 95064, USA

REBECCA A. W. ELSON

Institute of Astronomy, Cambridge CBS OHA, England

and

\section{THE MEDIUM DEEP SURVEY TEAM:}

R. E. GRIFFITHS, R. S. ELLIS, G. GILMORE, R. F. GREEN, J. P. HUCHRA, G. D. ILLINGWORTH, D. C. KOO, K. RATNATUNGA, A. TYSON AND R. A. WINDHORST

\begin{abstract}
Abotract.
We summarise the first year of operation of the Medium Deep Survey - a key project of the HST. Two fields in the LMC are discuseed and some preliminary ecientific results presented. We aleo comment on image deconvolution for the extragalactic fielde observed a part of the Medium Deep Survey.
\end{abstract}

\section{Introduction}

The Medium Deep Survey (MDS) is a key project of the Hubble Space Telescope (HST) using the 'parallel mode' of operation. While one instrument is looking at the target, an unused camera can be acquiring an image, several arcminutes away, in parallel to the primary target. This mode of operation, which began in January 1992, greatly increases the efficiency of the HST and allows a variety of scientific topics to be addressed. These topics include star counts in the Galactic halo, measuring the stellar luminosity function to fainter limits, determination of faint galaxy morphology and scale lengths, serendipitous discoveries of gravitational lenses and many more. MDS observations are about $30-50$ mins on average, although some are significantly longer. Follow-up ground based spectroscopic observations are being carried out using various large telescopes. Eventually these observations will form a large database suitable for the statistical analysis of random fields.

Roughly 80 different fields have been observed using the Wide Field Camera, Planetary Camera and the Faint Object Camera. Many of these fields are only single orbit which makes the removal of cosmic rays problematic. We now highlight some preliminary scientific results.

\section{Stellar Populations in the LMC}

Two fields located near the bar of the LMC, $\sim 10$ arcmin from the 30 Doradus nebula, have been observed. These WFC fields are 2.7 arcmin on a side. Field 1 was observed on 1992 June 6, and Field 2 on 1992 September 15.

After initial data reduction the images were cleaned of $\sim 3 \times 10^{4}$ cosmic rays. In the case of Field 2, where there were three independent exposures in the F785LP filter, we also median-filtered the images. This gives much more satisfactory results than simply cleaning individual images. We used DAOPHOT to automatically find 


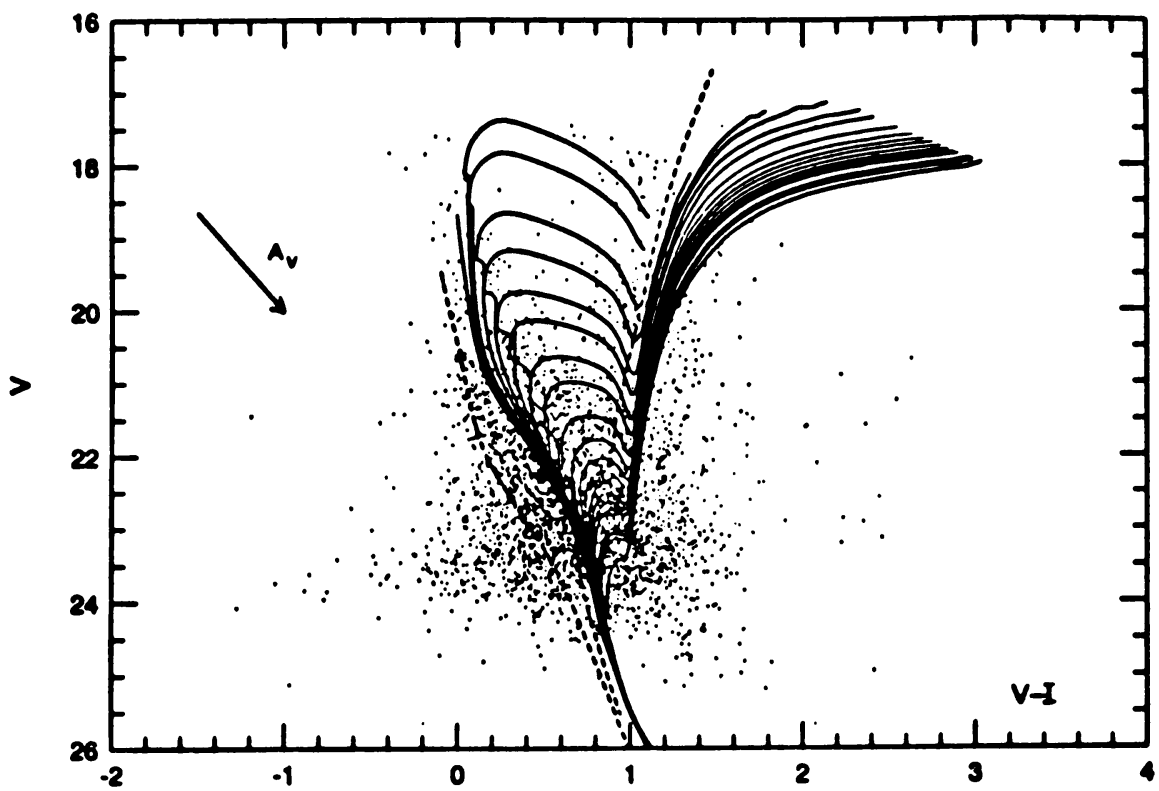

Fig. 1. Color-magnitude diagram for the Sept 15th field, containing about 5000 atars with magnitudes $18<V<26$. Stars brighter than $V=18$ are saturated. Typical ground-based observations of the LMC typically reach to $V \sim 22$. Superposed on the data we show the Yale isochrones for $Z=0.001$ and $Y=0.3$ (solid curves), ranging in age from $150 \mathrm{Myr}$ to $20 \mathrm{Gyr}$. We also give a low-metallicity isochrone $\left(Z=1 \times 10^{-5}\right)$ for $20 \mathrm{Gyr}$ and the zero age main sequence (dashed curves). The isochrones have been reddened by $E(V-I)=$ 0.15 , and converted to apparent magnitude using a distance of $52 \mathrm{kpc}$.

otars, and to perform aperture photometry on the cleaned (and in the case of Field 2 , coadded) images. The final lists of stars have positions which agree, between the F555W and F785LP images, to better than 2 pixels ( 0.2 arcsec).

Because of spherical aberration, the HST psf extends to a radius of about 3 arcsec (Burrows et al. 1991). Thus, even in fields which are only moderately crowded, such as those observed here, the psfs of individual stars overlap. An aperture large enough to include the total light of a star is likely to contain one or more neighbouring stars as well. We have therefore chosen to measure only the light in the cores of the stellar images, and apply aperture corrections to account for the light outside the core. To convert the core magnitudes to total magnitudes, we determined aperture corrections from a grid of $7 \times 7$ simulated point-spread functions for each chip and filter, constructed using TINYTIM (v2.1) (Krist 1992). Zero point offets were calculated from the WFPC Science Verification Report (Faber 1991), which have an uncertainty of $\approx 0.06^{\mathrm{m}}$. These are then converted to the standard JohnsonCousins system using the transformation by Harris et al. (1991). We estimate the final uncertainty to be $0.07^{\mathrm{m}}$ in $V$ and $0.15^{\mathrm{m}}$ in $(V-I)$.

A colour-magnitude diagram for the Sept 15th field is shown in Fig. 1. Isochrone fitting suggests three distinct stellar populations - the main sequence, the giant branch and a blue low-metallicity population. Fig. 2 shows a histogram of the 


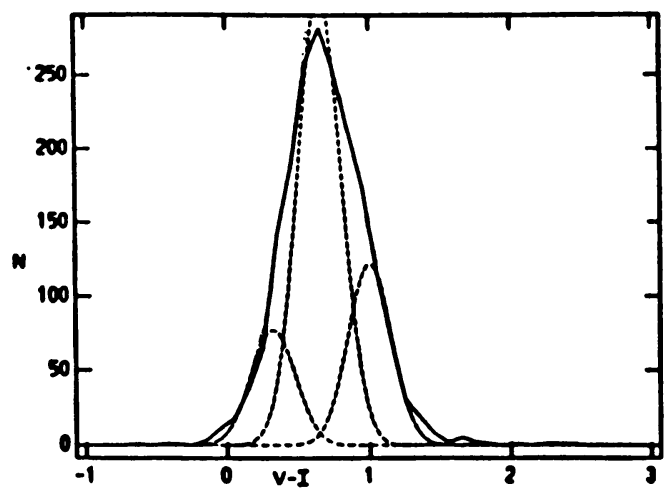

Fig. 2. Histogram for the Sept 15th field, showing the number of atare a a function of (V-I) color. The solid line represents the data. This distribution has been fit naing three Gausians, located at $(V-I)=0.32$ (a low metallicity population), 0.65 (the main equence) and 1.0 (the giant branch). The data are well fit by these three populations given our photometric error of $0.15^{\mathrm{m}}$ in $(\mathrm{V}-\mathrm{I})$.

number of stars as a function of colour. This suggests that the low-metallicity population has a $(V-I)$ colour of 0.32 .

\section{Galaxy Modeling and Image Deconvolution}

The majority of the MDS fields are at high galactic latitude and hence contain many faint galaxies. Some sort of galaxy modeling or image deconvolution is required before analysis can proceed. Work by Schade et al. (1992) suggests that the Memsy85 software may not be suitable for the deconvolution of galaxies with low $S / N$. We are currently testing the Richardson-Lucy and Lucy-Hook algorithms for deconvolution of simulated MDS galaxies. A new deconvolution method called OptMRL, based on Bayesian statistics, has beer. developed by Pina \& Puetter (1992). OptMRL employs two new techniques - spatially uncorrelated residuals and the image processing equivalent of Occam's razor. Although this method shows promise it has not yet become available for testing. An alternative approach we are also investigating is maximum likelihood fitting of simple model galaxies to the MDS data.

\section{References}

Burrows, C. J., et al. 1991, ApJ, 369, L21

Faber, S., 1991, WFPC Science Verification Report, STScI

Harris, H. C., et al. 1991, AJ, 101, 677

Krist, J., 1992, TINYTIM Users Manual, STScI

Pina, R. K., \& Puetter, R. C. 1992, PASP, 104, 1096

Schade, D. J., et al. 1992, AAS meeting 181, accepted 